\title{
Caracterización sociodemográfica y principales afecciones físicas y psicológicas del cuidador informal
}

\author{
Sociodemographic characterization and main physical and psychological affections of the informal caregiver \\ Caracterização sociodemográfica e principais condições físicas e psicológicas do cuidador informal
}

DOI: https://doi.org/10.21803/pensam.v11i21-1.265

\begin{abstract}
Lina María Ortiz Vargas
http://orcid.org/0000-0002-0218-7791

Carolina Franco Ramírez

http://orcid.org/0000-0003-3774-0400
\end{abstract}

Juan David Ortiz Polo

https://orcid.org/0000-0002-3506-435X

\section{¿Cómo citar este artículo?}

Ortiz, L., Franco, C. \& Ortiz, J. (2018). Caracterización sociodemográfica y principales afecciones físicas y psicológicas del cuidador informal. Pensamiento Americano, 11(22), 66-77. DOI: https://doi.org/10.21803/pensam.v17i21-1.265

\section{Resumen}

El presente es un artículo de revisión, en el que se pretende caracterizar socio demográficamente las principales afecciones físicas y psicológicas que sufre el cuidador informal; debido a diversas problemáticas que se presentan, esta población está en aumento, sin una adecuada preparación, y apoyo, lo cual conlleva a nuevas patologías. La metodología empleada fue una revisión documental, donde se evaluaron 64 artículos, los cuales tuvieron varios filtros según los criterios de elegibilidad. Posterior a esto, se realizó la validación de dichos artículos utilizando la escala de Jadad y las listas de chequeo STROBE, PRISMA, COREQ; se evaluaron correctamente 31 artículos en dos idiomas; 6 en inglés y 25 en español. Se pudo concluir que los $\mathrm{Cl}$, en su mayoría mujeres; requieren una red de apoyo extensa, bien sea familiar, que permita salirse de su rol de cuidadora y retomar el rol que tenían en la sociedad; además del apoyo dirigido por entidades gubernamentales. Como resultados se halló que los C.I. son de mediana edad, casadas, con escolaridad básica. Su parentesco con el sujeto de cuidado es en primer grado. Se evidenció de igual manera, que los hombres a pesar de no ejercer habitualmente este rol son quienes presentan más rápido agotamiento emocional.

\section{Palabras Clave: Cuidador informal, agotamiento.}

\begin{abstract}
In this review article, it is intended to characterize demographically, the main physical and psychological conditions suffered by the informal caregiver; due to various problems that arise, this population is increasing, without adequate preparation, and support, which leads to new pathologies. The methodology used was a documentary review, where 64 papers were evaluated, which had several filters according to the eligibility criteria. After this, the validation of these articles was carried out using the Jadad scale and the STROBE, PRISMA, COREQ checklists; 31 articles in two languages were correctly evaluated; 6 in English and 25 in Spanish. It could be concluded that IC, mostly women; they require an extensive support network, whether familial, that allows them to get out of their role as caregiver and resume their role in society; in addition to the support directed by government entities. It was found that the C.I., are middle-aged, married, with basic schooling. Her/his kinship with the subject of care is in the first degree. It was evidenced in the same way that men, despite not usually exercising such a role, they are ones who present the fastest emotional exhaustion.
\end{abstract}

Key words: Informal caregiver, exhaustion. 


\section{Resumo:}

O presente artigo de revisão pretende caracterizar sóciodemograficamente as principais condições físicas e psicológicas que sofrem o cuidador informal. Devido a diversas problemáticas que se apresentam, esta população está em aumento sem uma adequada preparação e apoio, o que leva a novas patologias. A metodologia utilizada foi a revisão documental onde foram avaliados 64 artigos, os quais tiveram vários filtros segundo os critérios de elegibilidade. Posterior a isso, foi realizada uma validação dos artigos utilizando a escala de Jadad e as listas de verificação STROBE, PRISMA, COREQ. Foram avaliados corretamente 31 artigos em dois idiomas; 6 em inglês e 25 em espanhol. A conclusão foi a de que os Cuidadores Informais (Cl), em sua maioria mulheres, requerem uma rede de apoio muito extensa, bem seja familiar, que permita a saída do papel de cuidador e o retorno ao papel anteriormente dado pela sociedade, aparte do apoio dado por entidades governamentais. Como resultado, então, temos que os $\mathrm{Cl}$ são indivíduos de meia idade, casados, com escolaridade básica. Seu parentesco com o sujeito é de primeiro grau. Se evidenciou, de igual forma, que os homens - a pesar de no exercer habitualmente este papel - são quem apresentam mais rápidos sinais de esgotamento emocional.

Palavras-chave: cuidador informal, esgotamento

\section{Perfil}

Lina María Ortiz Vargas

Ph.D Psicología.

en Neuropsicología y Educación. Imortiz@unac.edu.co

\section{Perfil}

Enfermera profesional

cafranco@unac.edu.co

\section{Perfil}

Enfermero Profesional. Corporación Universitaria Adventista juortiz@unac.edu.co 


\section{Problema o Necesidad}

L

os Cuidadores Informales (C.I), se han consolidado en la sociedad como las personas encargadas de brindar apoyo a los individuos que por algún motivo requieren de este; si bien es cierto, existe una profesión idónea para realizar estas actividades de cuidado, no es fácil acceder a estos; por esta razón, los C.I a pesar de no tener conocimientos científicos para realizarlos deben empoderarse de este rol y asumir la carga que esto conlleva.

Además, se ve incrementada la necesidad de estos C.I, puesto que la sociedad se encuentra en una transición poblacional, en donde el aumento de la población adulta es cada vez mayor, y a su vez la aparición de enfermedades crónicas que tienen como consecuencia la aparición de eventos incapacitantes en quienes las sufren.

\section{Presentación epistemológica del cuida- dor informal}

\subsection{Introducción}

El cuidado, ha sido definido por Achury (2006), como una característica innata requerida por todo ser humano, desde su nacimiento hasta su muerte; el cual, desde sus orígenes se brindó de manera empírica; en sus inicios las madres, abuelas y matronas eran designadas implícitamente por la sociedad a realizar actividades de cuidado. En el transcurrir natural de la vida, personas que no se podían valer por sí mismas o afectadas a causa de eventos particulares como las guerras, necesitaron también de una ayuda externa que les permitiera realizar por sí mismos el proceso de recuperar su autonomía. Ante todos estos hechos, surge la necesidad de personas con una formación enfocada a brindar un cuidado más idóneo que diera respuestas a estas problemáticas.

Sobre este tema la teórica Virginia Henderson afirma que:

Las enfermeras y los enfermeros tienen la función principal de atender a las personas enfermas o sanas para llevar a cabo las actividades que colaboran con su recuperación o bien a evitar que padezcan cuando se acerca su hora final. Los cuidados de enfermería son un servicio que compensan la falta de fuerza, voluntad o conocimiento, dependiendo del trastorno (Burgos \& Paravic, 2009).

Aunque exista una profesión encargada directamente de estas actividades de cuidado; hay diferentes circunstancias como lo menciona la Organización Mundial de la Salud (OMS) (Organización Mundial de la Salud, 2018) ᄀлson el aumento de enfermedades crónicas no transmisibles e incapacitantes y la pérdida de autonomía de la población adulta, que conllevan a que personas no profesionales, ejerzan como cuidadores informales; esto se puede ver evidenciado en el ASIS de Colombia (MINSALUD, 2016) en el cual se indica la inversión de la pirámide poblacional, teniendo como consecuencia mayores alteraciones en la salud y demanda de cuidado de la población adulta. Del Valle, Hernández y Zúñiga (2014), argumentan que las personas que toman este rol deben asumir esta responsabilidad como una obligación moral frente a la sociedad, lo que conlleva a un desgaste emocional, físico y económico.

\subsection{Planteamiento del problema}

Según la real academia española, define la palabra cuidar como poner en diligencia, atención y solicitud la atención de algo, 
pero al profundizar más este término se encontrará que la acción de cuidar, contiene otros matices, el cual resulta siendo un término bastante amplio, Como Siles y Solano (2007) lo afirman, este término contiene unas esencias, esto hace referencia a sus características, propiedades y relaciones más profundas, debido a que la acción de cuidar ha evolucionado a través del tiempo, y ha sido influenciado por el contexto social, la religión y el avance científico.

Achury (2006), indica que a través de la historia se ha evidenciado que la naturaleza del hombre es una constante preocupación por mantener un estado de salud óptimo que le permita sobrevivir, y ante la falta de dicha salud, nació la búsqueda de una llamada cura para recuperar esta, esto hizo que existieran diferentes tipos de acciones las cuales fueron llamadas cuidado.

Durante la historia del cuidado en las diferentes épocas y sociedades se pudo identificar cómo estas tareas eran delegadas a ciertas personas; por ejemplo, desde la antigüedad, las acciones de cuidado estaban relacionadas como lo menciona Achury (2006) en su publicación Historia del cuidado con el hecho de mantener la vida y el bienestar; razón por la cual, en esta época el cuidar estaba atribuido al rol de madre, puesto que eran estas quienes brindaban a sus familias desde el nacimiento hasta su muerte, las enseñanzas para mantener la salud y curar en caso de enfermedad. Durante la edad media esta labor estaba asignada a mujeres consagradas y eran enfocadas en su mayoría a curar en la iglesia o en el hospital a los más desvalidos. Ya a partir del siglo XIX, se encuentra la necesidad de entrenar a las cuidadoras y gracias a la aparición de Florence Nigthingale, se comienza a implementar el entrenamiento en el cuidado como una práctica basada en conocimientos técnicos.
A partir de esta evolución fundamentada en la formación técnica y la clasificación del cuidado en la labor de enfermería, surgió la necesidad de crear una disciplina que diera a esta ocupación un cuerpo justificado en conocimientos, no solamente técnicos y prácticos sino también teóricos; González, Arras y Moriel (2012) afirman que por tal razón emerge enfermería como disciplina con su propio método científico, el Proceso de Atención en Enfermería (PAE) y su razón de ser bien definida que es el cuidado.

Esta profesionalización del cuidado no extinguió la ocupación milenaria confiada a madres y a personas ajenas a una formación técnica a brindar acciones encaminadas al bienestar y protección de quienes más lo necesitan; es por esta razón, que aún en la actualidad, a pesar de existir una profesión específica para el cuidado coexisten los Ilamados cuidadores informales.

Se denominan cuidadores informales, de ahora en adelante C.I para este texto, a las personas que realizan la tarea de cuidado de personas enfermas, discapacitadas o ancianas, que no pueden valerse por sí mismas para la realización de actividades de la vida diaria, Como lo menciona Ruiz y Moya ( 2012) que en muchas ocasiones estas personas son designadas sin haber manifestado explícitamente su deseo hacerlo, lo cual conlleva a que no reciban esta tarea de forma adecuada, produciendo una sobrecarga hacia la función de cuidadores informales.

Si bien todos requieren de cuidados básicos, existen algunas personas que, por sus condiciones de salud o sus características, por el envejecimiento natural demandan asistencia de un cuidador; En su publicación Bayarre Vea (2017) demuestra que en la mayoría de los países de América latina, la pirámide poblacional ha tenido un cambio

Pensamiento Americano Vol. 11 (22) • 2018 • Julio-Diciembre · Corporación Universitaria Americana • Barranquilla, Colombia • ISSN: $2027-2448$. 
en su estructura llegando a aumentar cada vez más la población de adultos mayores, razón por la cual, puede existir dependencia y sobrecarga a un único individuo.

Sumado esto, Ávila y Vergara (2014), hacen referencia que el aumento de las enfermedades crónicas no transmisibles en sus etapas de mayor incapacidad, junto con el aumento de la población senil, disminuye la capacidad de autocuidado de estas personas, lo que conlleva a la necesidad de requerir C.I., produciendo en estos, desgaste por las demandas físicas y emocionales que esta labor requiere.

De igual manera, en la población infantil se presenta esta misma problemática, como lo menciona Barrera, Sánchez y Carrillo (2013) el desgaste físico y psicológico de los padres afecta su calidad de vida. Espinoza y Jofre (2012) mencionan en su publicación que la sobrecarga implica a los cuidadores problemas físicos, mentales, económicos y altera de igual manera el desarrollo de actividades de la vida cotidiana; alteraciones que son llamadas en los cuidadores profesionales, "síndrome Burnout", definido en 1976 por Maslach y Jackson como el agotamiento emocional, despersonalización y poca realización personal.

Barrera, Sánchez y Carrillo (2013) afirma que la demanda de tiempo que comprende ser C.I., afecta la condición económica, debido a que por las extenuantes jornadas que pasan con su sujeto de cuidado, se ven en la necesidad de abandonar su trabajo o disminuir su tiempo laboral, de esta manera los recursos económicos se ven perjudicados y sumado a esto, se encuentra el incremento de los gastos que debe de realizar esta persona para la manutención. A partir de esto, se puede evidenciar en Colombia como los C.I. no son beneficiados de ayudas públicas que les permitan menguar sus cargas.
Se puede observar a través de diferentes publicaciones como algunos países abordan el tema de cuidadores informales de una forma más incluyente en sus políticas públicas; por ejemplo, en España la consejería Andaluza de salud y bienestar establece entre sus prioridades la mejora de la atención a las familias y a las personas cuidadoras, basándose en el decreto de apoyo a las familias andaluzas. Flores y Seguel (2016), evidencia que en América latina los cuidadores informales manifiestan no recibir el suficiente apoyo, específicamente en Chile donde los cuidadores informales perciben un bajo nivel de apoyo social afectivo y de confianza, argumentando que no existe un nivel de información y comunicación para expresar sus sentimientos derivados de los problemas que se generan al cuidar.

A nivel local, el ministerio de salud de Colombia (Congreso de Colombia, 2013) expidió en el 2013 la ley 1616 enfocada hacia la salud mental la cual en su artículo 9, obliga a las administradoras de riesgos laborales a realizar actividades de promoción y prevención para la salud mental; con respecto a los C.I. no existe una ley que los ampare, el único acercamiento que se ha tenido han sido proyectos de ley que no han avanzado y por ende hay un desamparo hacia estos en términos legales.

Ante este panorama tan desfavorecedor de los cuidadores informales se hace pertinente realizar una revisión bibliográfica que permita determinar quiénes son y cuáles son sus principales características, además de dar a conocer ¿Son los C.I. las personas idóneas para soportar el trabajo de la enfermería profesional?

\subsection{Pregunta}

¿Cuáles son las principales características sociodemográficas y sus principales afectaciones físicas y psicológicas del cuidador informal? 


\subsection{Objetivo}

Realizar una caracterización sociodemográfica de los cuidadores informales y sus principales afectaciones físicas y psicológicas.

\subsection{Justificación}

La revisión de diferentes artículos posibilita conocer el estado de arte actual de los temas de interés; como es el caso; además permite ampliar los conocimientos sobre una situación emergente en la sociedad que son cuidadores informales, sumado a esto, brinda a los investigadores el poder identificar varios aspectos relacionados con dicha temática, ya sea conocer quiénes son las personas que mayormente desempeñan dicho rol y sus diferentes características.

Con esta exploración bibliográfica se pretende identificar las principales características sociodemográficas, físicas y psicológicas de los cuidadores informales, identificando que factores influyen negativamente en su calidad de vida, esto ayudara a establecer un conocimiento compacto sobre estos aspectos, con la finalidad de dar a conocerlos y que es- tos puedan ser usados para generar soluciones que garanticen un adecuado manejo en la salud de los ya mencionados cuidadores informales.

\section{Metodología}

Se llevó a cabo una revisión documental, el proceso de búsqueda permitió revisar 64 estudios y a partir de esto, se realizaron varios filtros que permitieron descartar los artículos según los criterios de elegibilidad establecidos; el primero excluyó 10 artículos previos al 2012; para el segundo filtro se contó con 54 estudios, de los cuales 8 no cumplían puesto que trataban el tema de cuidadores formales; de los 46 artículos restantes 8 fueron descartados ya que se centraban en la patología el sujeto de cuidado. Finalmente 38 artículos cumplieron con los criterios definidos. Posterior a esto, se realizó la validación de dichos artículos utilizando la escala de Jadad y las listas de chequeo STROBE, PRISMA y COREQ. Estas herramientas permitieron descartar artículos que su validez no era adecuada para su uso y evaluaron correctamente 31 artículos en dos idiomas; 6 de ellos en inglés y los 25 restantes en español. 3.

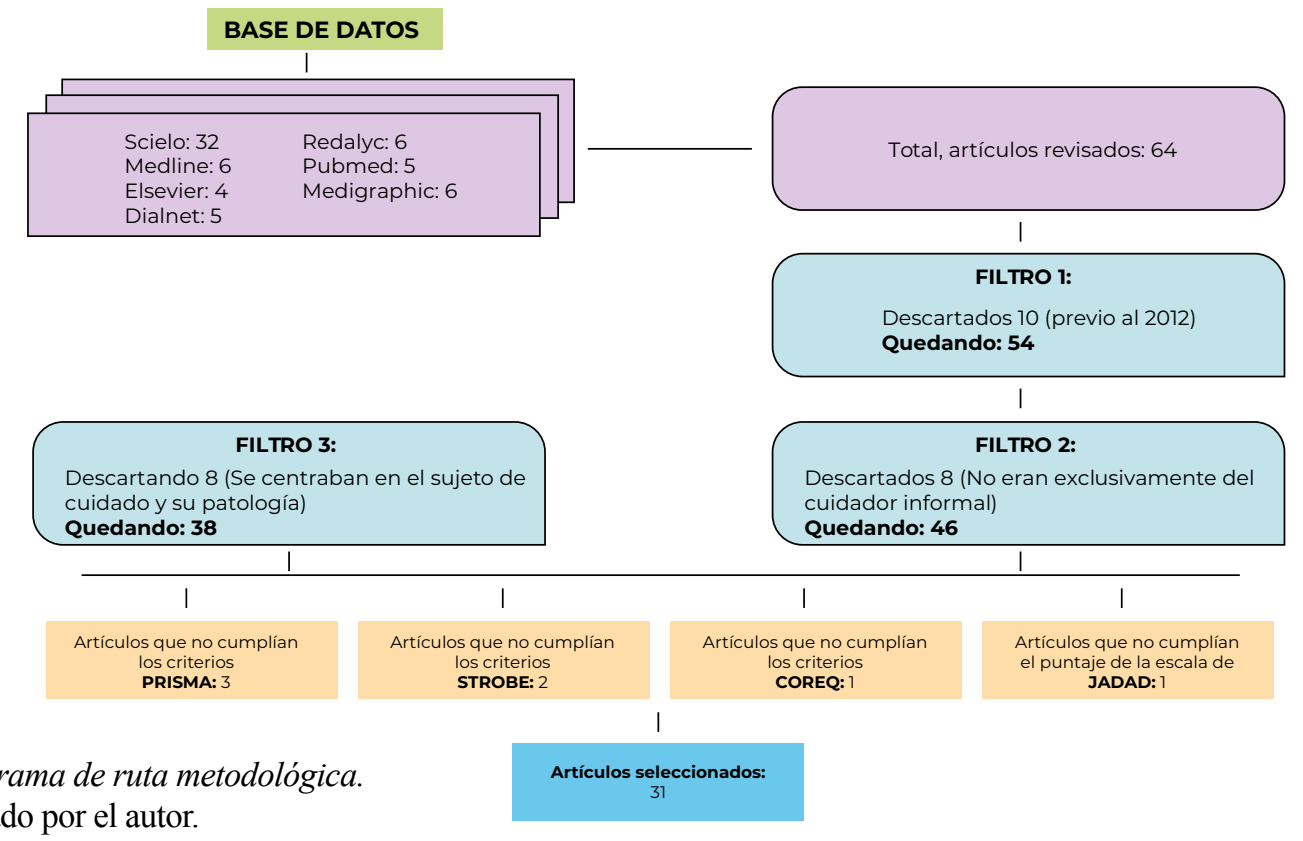

Gráfica 1. Diagrama de ruta metodológica.

Fuente: Elaborado por el autor. 


\section{Resultados}

La revisión bibliográfica acerca de cuidadores informales, permitió identificar ciertas características de importancia que cabe la pena resaltar; de estos 31 artículos revisados tanto en inglés (6) como en español (25), se pudo realizar la caracterización de las rasgos sociodemográficas, psicológicas y físicas de los cuidadores informales puesto que en su mayoría estos tratan dichas temáticas; por tal motivo los resultados brindados en la discusión se hicieron con base a esta información obtenida.

Se revisaron diferentes bases de datos para obtener los diferentes artículos, las cuales fueron Scielo. Pubmed, Elsevier, Medigraphic, Redalyc, Dialnet, además se tuvieron en cuenta estudios de diferentes países como España, Brasil, Colombia, Canadá, Chile, México, Cuba, India, Venezuela y Bélgica. (Tabla 2).

A partir de la revisión de los artículos, se determinaron diferentes variables de los cuidadores informales, los cuales incluían: sexo, edad, parentesco con el sujeto de cuidado, nivel educativo, estado civil, ocupación, ingresos económicos, razón por la cual eran cuidadores informales, el tiempo de cuidado y la necesidad de recibir de apoyo de otra persona para realizar la labor. (Tabla 3).

De igual manera, los artículos acerca de cuidadores informales mencionan aspectos del sujeto de cuidado, como su edad, sexo y afección principal (Tabla 4).
Tabla 1.

Cantidad de artículos según fuentes de información

\begin{tabular}{lc}
\hline Bases de datos & Cantidad de artículos \\
\hline Scielo & 27 \\
Pubmed & 3 \\
Elsevier & 3 \\
Medigraphic & 2 \\
Resalyc & 3 \\
Dialnet & 1 \\
\hline
\end{tabular}

Fuente: Elaboración del autor

Tabla 2.

Cantidad de artículos según país de publicación

\begin{tabular}{lc}
\hline Países & Cantidad de artículos \\
\hline España & 9 \\
Brasil & 7 \\
Colombia & 8 \\
Canadá & 1 \\
Chile & 5 \\
México & 5 \\
Cuba & 1 \\
India & 1 \\
Venezuela & 1 \\
Bélgica & 1 \\
\hline
\end{tabular}

Fuente: Elaboración del autor 
Tabla 3.

Resultados de variable C.I. según el número de artículos

\section{Variables}

Sexo

Sexo

Edad

\begin{tabular}{|l} 
\\
\hline Parentesco con el \\
sujeto de cuidado
\end{tabular}

Nivel Educativo

Estado civil

Razón de cuidado

Tiempo de cuidado en h/día

Tiempo de cuidado en meses

Apoyo de terceros

Afecciones

$\begin{array}{ll}\text { Hombre } & 18 \\ \text { Mujer } & 30 \\ \text { Juventud (14-26 años) } & 7\end{array}$

Adultez (27-59 años) 24

Vejez (>60 años) $\quad 10$

Hijos 26

Hermanos 11

Padres 4

Cónyuges 22

Básico 20

Medio 10

Superior $\quad 8$

Soltero 14

Casado 25

Divorciado 8

Encargado del hogar $\quad 21$

Empleado 13

Altos 3

Suficientes 6

Bajos 17

Iniciativa propia $\quad 16$

Impuesto 3

$<12 \mathrm{~h} /$ día $\quad 7$

$>12 \mathrm{~h} /$ día $\quad 18$

$<12$ meses 4

$>12$ meses $\quad 19$

$\mathrm{Si}$

No 21

Físicas 17

$\begin{array}{ll}\text { Psicológicas } & 27\end{array}$ 
Tabla 4.

Resultados de variable del sujeto de cuidado según el número de artículos

\begin{tabular}{llc}
\hline Variables & & \multicolumn{2}{c}{$\begin{array}{c}\text { Cantidad de artículos } \\
\text { lo que mencionan }\end{array}$} \\
\hline Sexo & Hombre & 11 \\
& Mujer & 7 \\
Edad & Juventud (14-26 años) & 3 \\
& Adultez (27-59 años) & 4 \\
& Vejez (>60 años) & 22 \\
\hline \multirow{2}{*}{ Afecciones } & Crónicas & 30 \\
\hline
\end{tabular}

Fuente: Elaboración del autor

Además, otro aspecto relevante que se halló fue las principales características de los cuidadores informales que mencionaban los artículos revisados, como se puede evidenciar en la siguiente tabla

Tabla 5.

Resultados según variables de caracterización del C.I.

Caracterización del cuidador informal

\begin{tabular}{lll}
\hline Variables & Mujer & $\begin{array}{c}\text { Cantidad de artículos } \\
\text { que lo mencionan }\end{array}$ \\
\hline Sexo & Adultez (27-59 años) & 30 \\
Edad & Hija & 24 \\
Parentesco & Hermanos & 26 \\
Estado civil & Casada & 25 \\
Nivel Educativo & Básico & 20 \\
Ocupación & Encargada del hogar & 21 \\
Ingresos económicos & Bajos & 17 \\
Razón de cuidado & Iniciativa propia & 18 \\
Tiempo de cuidado en h/día & $>12 h /$ día & 19 \\
Tiempo de cuidado en meses & $>12$ meses & 21 \\
\hline Apoyo de terceros & & \\
\hline
\end{tabular}

Fuente: Elaboración del autor

Otro descubrimiento después de revisar los artículos fue las afecciones que sufrían los cuidadores informales por la realización de esta labor, lo cual se puede evidenciar en la siguiente tabla
Tabla 6.

Resultados de según las afecciones del C.I.

\section{Afecciones}

\begin{tabular}{|c|c|c|}
\hline \multirow{3}{*}{$\begin{array}{l}\text { Cantidad de } \\
\text { artículos que } \\
\text { lo mencionan }\end{array}$} & Físicas & Psicológicas \\
\hline & $\begin{array}{l}\text { Hipertensión arterial } \\
\text { hipercoleterolemia } \\
\text { gastritis }\end{array}$ & $\begin{array}{l}\text { Estrés crónico, } \\
\text { crisis de ansiedad, } \\
\text { agotamiento } \\
\text { emocional, } \\
\text { síndrome de } \\
\text { Burnout }\end{array}$ \\
\hline & 17 & 27 \\
\hline
\end{tabular}

Fuente: Elaboración del autor

Sea válido, que, a partir de la revisión bibliográfica, se pudieron hallar datos relevantes, y que, aunque no fueran parte del objetivo de la investigación, se consideraron importantes dentro de la temática; los cuales fueron las características del sujeto de cuidado, dentro de estas se presentaron algunas de las siguientes características: la edad en promedio fue de 70 años, género masculino, con presencia de enfermedades crónicas, y el vínculo con el cuidador en primer grado

Tabla 7.

Resultados de según variables de caracterización del sujeto cuidado

\section{Características del sujeto de cuidado}

Cantidad de artículos
que lo mencionan

\begin{tabular}{lll} 
Edad & Vejez (>60 años) & 22 \\
Sexo & Hombre & 12 \\
Afecciones & Crónicas & 30 \\
\hline
\end{tabular}

Fuente: Elaboración del autor 


\section{Discusión}

En relación con la población en estudio es relevante conocer de estos las siguientes características:

Los cuidadores informales, según Ávila y Vergara (2014) son un fenómeno en aumento de la sociedad actual, debido a una diversidad de factores socioculturales, como lo puede ser la actual situación económica, el aumento de la población senil, el incremento de las enfermedades crónicas, la insuficiente capacidad de respuesta por parte de las instituciones de salud, entre otros factores; lo cual ha obligado a personal sin formación adecuada, ni capacitación, a asumir este rol.

Existen hombres encargados del rol de cuidadores informales, pero siempre en menor medida que las mujeres, además, según Margaret Penning y Wu (2015), "en el género masculino, hay un mayor agotamiento emocional, y de forma más acelerada" (p.56), al estar encargados de realizar este tipo de cuidados, en comparación con el género femenino lo cual conlleva, a que los mismos, presenten mayores dificultades a nivel laboral, personal, y familiar, hasta el punto de abandonar su rol como cuidadores.

Se demostró que el solo hecho de ser un cuidador informal, es factor predisponente para desarrollar afecciones mentales, como lo expone Crespo y Fernández (2015) en su estudio "Resiliencia en cuidadores familiares de personas mayores dependientes" entre las más significativas se encontraron las siguientes: Estrés crónico, ansiedad, depresión, autopercepción negativa, aislamiento, disminución de la libido, agotamiento emocional, autoacusación, entre otros. Lo cual se puede interpretar, a partir los estudios analizados, en donde esta situación puede ser causada, debido a los largos periodos que demanda este tipo de atención, con un promedio de 18 horas al día, durante 46 meses.

Este tiempo también influye en la salud física, en el desarrollo de enfermedades crónicas, debido al alto índice de estrés al cual están expuestas estas personas, algunas de esas enfermedades fueron: Enfermedades cardiovasculares (hipertensión, isquemia), hipercolesterolemia, gastritis crónica, trastornos del sueño, trastornos alimenticios, y afectación en el sistema inmune.

Lo anterior conlleva, en algunos casos, a generar dependencia de tratamiento farmacológico, por parte de los cuidadores, lo cual puede influir en que en un futuro los cuidadores pasen a ser sujetos de cuidado.

Otro aspecto relevante hallado fue, la afectación a la situación económica del cuidador, lo cual se debe a que una gran mayoría no recibe ningún tipo de remuneración por el trabajo hecho, por otra parte, se encontró que un alto porcentaje son de estrato socioeconómico bajo, además este tipo de atención a enfermedades crónicas implica un alto gasto económico, tanto por el alto costo de los tratamientos, como por la duración de los mismos. Además de lo anterior mencionado, los recursos del cuidador son insuficientes, debido a que una gran mayoría no cuenta con un trabajo, y los que tienen, lo pierden por el alto índice de ausentismo laboral, y en algunos casos en donde llegan a renunciar.

\section{Conclusiones}

La revisión de artículos acerca de cuidadores informales permitió inferir algunas características sociodemográficas de estos. Encontrándose que quienes mayormente realizan acciones de cuidado son mujeres adultas, casadas; que además de esta responsabilidad de cuidar, están a cargo de las labores

Pensamiento Americano Vol. 11 (22) • 2018 • Julio-Diciembre • Corporación Universitaria Americana • Barranquilla, Colombia • ISSN: $2027-2448$. 
propias del hogar como principal ocupación. De igual manera, se encontró que cuentan con un nivel educativo básico y que dada la ausencia de un empleo remunerado estas cuidadoras, no poseen un ingreso económico estable, provocando que los recursos sean insuficientes para suplir sus propias necesidades y las del sujeto de cuidado.

Asimismo, se encontró que las cuidadoras informales son por lo general, hijas del sujeto de cuidado y su razón para realizar esta acción no es impuesta; por el contrario, se evidenció que su motivo para acompañar a esta persona que requiere de su asistencia es voluntad propia. A pesar de esto, se hace necesaria la ayuda de un tercero que permita aliviar un poco la carga propina por la persona cuidada, que según lo hallado son adultos mayores en estado de cronicidad y como bien se sabe, las obligaciones con respecto a su cuidado son considerables y no cuentan con un apoyo para realizarlas.

Por tal motivo, estas mujeres pasan más de 12 horas durante el día, por un periodo prolongado, velando por el bienestar de su pariente; lo cual, representa en ellas alteraciones de su salud; como es la mayoría de los casos, aparecen más afecciones psicológicas dadas por el estrés crónico, ansiedad, agotamiento emocional llevando finalmente a un síndrome de Burnout y en una menor medida afecciones físicas. Ello debido al poco cuidado de sí mismas, bien sea por falta de tiempo o de intención.

Se puede concluir que los cuidadores informales, en su mayoría mujeres; requieren de una red de apoyo extensa, bien sea familiar, que les permita por ciertos momentos salirse de su rol de cuidadora y retomar el rol que tenían en la sociedad; y un apoyo dirigido por entidades gubernamentales, que les proporcione la manera de subsanar los insuficientes ingresos económicos a causa de no contar con un empleo remunerado. Esto permitirá que a futuro se disminuyan las afecciones físicas y psicológicas que conllevan ser cuidador; y de esta manera, la probabilidad que esa persona cuidadora se convierta en un sujeto de cuidado. 


\section{Referencias}

Barrera, L., Sánchez, B. \& Carrillo, G. M. (2013). La carga del cuidado en cuidadores de niños con enfermedad crónica. Revista Cubana de Enfermería, 29(1).

Penning, M., \& Wu, Z. (2015). Caregiver stress and mental health: impact of caregiving relationship and gender. The gerontologist, 56.

Achury, D. M. (2006). La historia del cuidado y su influencia en la concepción y evolución de la enfermería. Investigación en Enfermería: Imagen y Desarrollo, 8.(1-2).

Ávila, J. \& Vergara, M. (2014). Calidad de vida en cuidadores de personas con enfermedad crónicas. Aquichan, 14(3).

Bayarre Vea, H. D. (2017). Múltiples perspectivas para el análisis del envejecimiento demográfico. Una necesidad en el ámbito sanitario contemporáneo. Revista Cubana de Salud Pública, 43(2).

Burgos Moreno, M. \& Paravic Klijn, T. (2009). Enfermería como profesión. Revista Cubana de Enfermería., 25(1-2).

Congreso de Colombia. (21 de enero de 2013). Ley de SaIud mental. [Ley 1616 de 2013]. DO: 48.680

Crespo, M. \& Fernández, V. (2015). Resiliencia en cuidadores familiares de personas mayores dependientes. Anales de psicología, 31(1).

Del Valle, M., Hernández, I. \& Zúñiga, M. (A 2014). Sobrecarga y Burnout en cuidadores informales del adulto mayor. Enfermería Universitaria, 12(1).

Espinoza, K. \& Jofre, V. (2012). Sobrecarga, apoyo social y autocuidado en cuidadores informales. Ciencia $y$ enfermería, 18(2).

Flores, E. \& Seguel, F. (2016). Functional social support in family caregivers of elderly adults with severe de- pendence. Invest Educ Enferm, 34(1).

González, E., Arras, A., \& Moriel, L. (2012). La profesionalización en enfermería: hacia una estrategia de cambio. El científico frente a la sociedad, 6(1).

MINSALUD. (2016). Analisis de situación en salud (ASIS Colombia,2016). Recuperado del Ministerio de Salud: https://www.minsalud.gov.co/sites/rid/Lists/BibliotecaDigital/RIDE/VS/ED/PSP/asis-colombia-2016.pdf

Organización Mundial de la Salud. (2018). Recuperado de Sitio web OMS: http://www.who.int/es/news-room/ fact-sheets/detail/noncommunicable-diseases

Ruiz, N. \& Moya, L. (2012). El cuidador informal: una vision actual. Revista de Motivación y Emoción, 22(30).

Siles, J. \& Solano, C. (2007). El origen fenomenológico del "cuidado" y la importancia del concepto de tiempo en la historia de enfermería. Cultura de los cuida$\operatorname{dos}(21)$. 